\title{
The uniform convergence of upwind schemes on layer-adapted meshes for a singularly perturbed Robin BVP
}

\author{
Quan Zheng, Fengxi Huang, Xiaoli Feng, Mengbin Han \\ College of Sciences, North China University of Technology, Beijing 100144, China \\ zhengq@ncut.edu.cn
}

\begin{abstract}
In this paper, we discuss the uniform convergence of the simple upwind scheme on the Shishkin mesh and the Bakhvalov-Shishkin mesh for solving a singularly perturbed Robin boundary value problem, and investigate the midpoint upwind scheme on the Shishkin mesh and the Bakhvalov-Shishkin mesh to achieve better uniform convergence. The elaborate $\varepsilon$ -uniform pointwise estimates are proved by using the comparison principle and barrier functions. The numerical experiments support the theoretical results for the schemes on the meshes.
\end{abstract}

Keywords-Singularly perturbed Robin BVP; simple upwind scheme; midpoint upwind scheme; layer-adapted mesh; uniform convergence

\section{Introduction}

Let us consider a singularly perturbed convection-diffusion Robin boundary value problem:

$$
\left\{\begin{array}{c}
L u \equiv-\varepsilon u^{\prime \prime}-b(x) u^{\prime}+c(x) u=f(x), x \in(0,1) \\
B_{0} u \equiv u(0)=A, B_{1} u \equiv u(1)+u^{\prime}(1)=B,
\end{array}\right.
$$

where $0<\varepsilon \ll 1$ is a small perturbation parameter, $A$ and $B$ are given constants, and function $b(x), c(x)$ and $f(x)$ are sufficiently smooth with $b(x) \geq \beta_{0}>\beta>0$ and $c(x) \geq 0$. Under these conditions, the singularly perturbed problem (1) has a unique solution with a boundary layer at $x=0$. Singularly perturbed problems arise in many branches of science and engineering such as modeling fluid flows and simulating semiconductor devices (see [1-4]). A wide variety of numerical methods, including the simple upwind scheme and the midpoint upwind scheme on layer-adapted meshes, were constructed to solve the problems in the past few decades (see [5-9]).

In this paper, the properties of the exact solution and the Shishkin mesh are introduced in section 2 . In section 3 , we discuss the simple upwind scheme on the Shishkin mesh for solving the singularly perturbed Robin BVP (1) and prove its $\varepsilon$-uniform pointwise convergence of order $O\left(N^{-1}\right)$ on the nodes in coarse part and $O\left(N^{-1} \ln N\right)$ on the nodes in fine part. In section 4, the simple upwind scheme on the Bakhvalov-Shishkin mesh, and the midpoint upwind scheme on the Shishkin mesh and the Bakhvalov-Shishkin mesh are studied to reach higher orders of uniform convergence. In section 5, several numerical examples support the elaborate error estimates.

\section{The Solution and the Mesh}

Lemma 1 (see [5]) For any positive integer $q>0$, if $u(x)$ is the solution of problem (1) with sufficiently smooth data then $u(x)$ can be decomposed as $u=S+E$, where the smooth part $S$ satisfies

$$
L S(x)=f(x) \text { and }\left|S^{(i)}(x)\right| \leq C, \quad 0 \leq i \leq q,
$$

while the part $E$ satisfies $L E(x)=0$,

and $E^{(i)}(x) \leq C \varepsilon^{-1} \exp \left(-\frac{\beta x}{\varepsilon}\right), 0 \leq i \leq q$.

Let $\tau=\min \left\{\frac{1}{2}, \frac{2 \varepsilon \ln N}{\beta}\right\}, N$ be an even positive number, and $\tau$ be the transition point, where $\varepsilon \leq N^{-1}$ as generally in practice. We have the Shishkin mesh:

$$
x(i)=\left\{\begin{array}{c}
\frac{2 \tau}{N} i, 0 \leq i \leq \frac{N}{2}, \\
\tau+\frac{2(1-\tau)\left(i-\frac{N}{2}\right)}{N}, \frac{N}{2} \leq i \leq N,
\end{array}\right.
$$

which is simply piecewise equidistant. Denoting $h_{i}=x_{i}-x_{i-1}$, we have

$$
\underset{i=1,2, \ldots, N / 2 .}{\text { Lemma }} \text { 2. } h_{i} \leq \frac{4 \varepsilon \ln N}{\beta N} \quad, \quad N^{-1} \leq h_{N / 2+i} \leq 2 N^{-1},
$$

Throughout the paper, $C$ is a generic positive constant that is independent of $\varepsilon$ and $h_{i}$, and note that $C$ can take different values at each occurrence, even in the same argument. 


\section{The Scheme and Its Estimate}

For the simple upwind scheme:

$$
\left\{\begin{array}{r}
L^{N} u_{i}^{N} \equiv-\varepsilon D^{+} D^{-} u_{i}^{N}-b_{i} D^{+} u_{i}^{N}+c_{i} u_{i}^{N}=f_{i} \\
\\
i=1,2, \ldots, N-1, \\
B_{0}^{N} u_{0}^{N} \equiv u_{0}^{N}=A, B_{1}^{N} u_{N}^{N} \equiv u_{N}^{N}+D^{-} u_{N}^{N}=B,
\end{array}\right.
$$

where

$$
D^{+} u_{i}^{N}=\frac{u_{i+1}^{N}-u_{i}^{N}}{h_{i+1}}, D^{-} u_{i}^{N}=\frac{u_{i}^{N}-u_{i-1}^{N}}{h_{i}}, D^{+} D^{-} u_{i}^{N}=\frac{2\left(D^{+} u_{i}^{N}-D^{-} u_{i}^{N}\right)}{h_{i+1}+h_{i}},
$$

we have

$$
\begin{aligned}
& \begin{aligned}
L^{N} u_{i}^{N} & =-\frac{2 \varepsilon}{\left(h_{i+1}+h_{i}\right) h_{i}} u_{i-1}^{N}+\left(\frac{2 \varepsilon}{h_{i+1} h_{i}}+\frac{b_{i}}{h_{i+1}}+c_{i}\right) u_{i}^{N}-\left(\frac{2 \varepsilon}{\left(h_{i+1}+h_{i}\right) h_{i+1}}+\frac{b_{i}}{h_{i+1}}\right) u_{i+1}^{N} \\
& =-r_{i}^{-} u_{i-1}^{N}+r_{i}^{c} u_{i}^{N}-r_{i}^{+} u_{i+1}^{N},
\end{aligned} \\
& \text { and } r_{i}^{-}, r_{i}^{c}, r_{i}^{+}>0,-r_{i}^{-}+r_{i}^{c}-r_{i}^{+}=c_{i} \geq 0, \quad i=1,2, \ldots, N-1 .
\end{aligned}
$$

Lemma 3. If grid function $v_{i}(x)$ and $w_{i}(x)$ satisfy $\mathrm{B}_{0}^{N} v_{0} \leq \mathrm{B}_{0}^{N} w_{0}, \quad \mathrm{~B}_{1}^{N} v_{N} \leq \mathrm{B}_{1}^{N} w_{N}$ and $L^{N} v_{i} \leq L^{N} w_{i}, i=1,2, \ldots, N-1$, then $v_{i}(x) \leq w_{i}(x), i=0,1,2, \ldots, N$, and the equation (3) has a unique solution.

Proof. It is proved by that the coefficient matrix associated with $L^{N}$ is an M-matrix.

By direct computation and Taylor formulas as usual, we have the following two lemmas.

$$
\begin{aligned}
& \text { Lemma 4. If } Z_{0}=1, Z_{i}=\prod_{j=1}^{i}\left(1+\frac{\beta h_{j}}{2 \varepsilon}\right), \\
& \qquad \text { then } L^{N} Z_{i} \geq \frac{C Z_{i}}{\max \left\{\varepsilon, h_{i+1}\right\}}, i=1,2, \ldots, N-1 .
\end{aligned}
$$

Lemma 5. $\left|L^{N}\left(u_{i}-u_{i}^{N}\right)\right| \leq C\left[\varepsilon \int_{x_{i-1}}^{x_{i+1}}\left|u^{\prime \prime \prime}(t)\right| d t+\int_{x_{i-1}}^{x_{i}}\left|u^{\prime \prime}(t)\right| d t\right]$.

As in the continuous case, decompose the numerical solution into the smooth part and the layer part by $u_{i}^{N}=S_{i}^{N}+E_{i}^{N}$, we have $L^{N} S_{i}^{N}=f_{i}, i=0,1,2, \ldots, N-1$, $B_{0}^{N} S_{0}^{N}=S(0)$,

$$
B_{1}^{N} S_{N}^{N} \equiv S_{N}^{N}+\frac{S_{N}^{N}-S_{N-1}^{N}}{h_{N}}=S(1)+S^{\prime}(1)
$$

and $L^{N} E_{i}^{N}=0, \quad i=0,1, \ldots, N-1, \quad B_{0}^{N} E_{0}^{N}=E(0)$,

$$
B_{1}^{N} E_{N}^{N} \equiv E_{N}^{N}+\frac{E_{N}^{N}-E_{N-1}^{N}}{h_{N}}=E(1)+E^{\prime}(1) .
$$

Therefore, the error can be estimated by

$$
\left|u_{i}-u_{i}^{N}\right| \leq\left|S_{i}-S_{i}^{N}\right|+\left|E_{i}-E_{i}^{N}\right| .
$$

For the smooth part, we have $\left|B_{0}^{N}\left(S_{0}-S_{0}^{N}\right)\right|=0$,

$$
\left|B_{1}^{N}\left(S_{N}-S_{N}^{N}\right)\right|=\left|B_{1}^{N} S_{N}-\left(B_{1} S\right)_{N}\right| \leq C N^{-1},
$$

$$
\left|L_{1}^{N}\left(S_{i}-S_{i}^{N}\right)\right|=\left|L_{1}^{N} S_{i}-\left(L_{1} S\right)_{i}\right| \leq C N^{-1},
$$

for $i=1,2, \ldots, N-1$, by Lemma 1 and Lemma 5. Setting $\omega_{i}=C N^{-1}\left(3-x_{i}\right)$ for all $i$, we have $L^{N} \omega_{i} \geq C N^{-1}$ $\geq\left|L^{N}\left(S_{i}-S_{i}^{N}\right)\right|$. By the discrete comparison principle, we get

$$
\left|S_{i}-S_{i}^{N}\right| \leq \omega_{i} \leq C N^{-1}, \quad i=0,1,2, \ldots, N .
$$

For the layer part, we have

Lemma 6. There exists a constant $C$ such that

$$
\left|E_{i}-E_{i}^{N}\right| \leq C N^{-1}, \quad i=N / 2, \ldots, N .
$$

Proof. By Lemma 1, we have $\left|B_{0}^{N} E_{0}^{N}\right|=|E(0)| \leq C$ and

$$
\begin{aligned}
\left|B_{1}^{N} E_{N}^{N}\right| & \leq|E(1)|+\left|E^{\prime}(1)\right| \leq C\left(\varepsilon^{-1}+1\right) e^{\frac{-\beta}{\varepsilon}} \leq C e^{\frac{-\beta}{2 \varepsilon}} \\
& =C \prod_{j=1}^{N} e^{\frac{-\beta h_{j}}{2 \varepsilon}} \leq C \prod_{j=1}^{N}\left(1+\frac{\beta h_{j}}{2 \varepsilon}\right) .
\end{aligned}
$$

Let $Y_{i}=C_{0} Z_{i}+C_{0} \frac{\beta}{2 \varepsilon} Z_{N}$ and $C_{0}$ to be sufficiently large, then $\quad B_{0}^{N} Y_{0} \geq C_{0} \geq\left|B_{0}^{N} E_{0}^{N}\right| \quad, \quad B_{1}^{N} Y_{N}=C_{0} Z_{N} \geq\left|B_{1}^{N} E_{N}^{N}\right| \quad$ and $L^{N} Y_{i} \geq 0=\left|L^{N} E_{i}^{N}\right|, \quad i=1,2, \ldots, N-1$. So, $Y_{i}$ is a discrete barrier function for $E_{i}^{N}$, and noting

$$
\begin{gathered}
\ln \prod_{j=1}^{N / 2}\left(1+\frac{\beta h_{j}}{2 \varepsilon}\right) \geq \sum_{j=1}^{N / 2}\left(\frac{\beta h_{j}}{2 \varepsilon}-\frac{1}{2}\left(\frac{\beta h_{j}}{2 \varepsilon}\right)^{2}\right) \\
\geq \frac{\beta x_{N / 2}}{2 \varepsilon}-\frac{2 \ln ^{2} N}{N} \geq \frac{\beta x_{N / 2}}{2 \varepsilon}-C, \\
\prod_{j=1}^{N / 2}\left(1+\frac{\beta h_{j}}{2 \varepsilon}\right)^{-1} \leq C e^{-\frac{\beta x_{N / 2}}{2 \varepsilon} \leq C N^{-1},} \\
\frac{\beta}{2 \varepsilon} \prod_{j=N / 2+1}^{N}\left(1+\frac{\beta h_{j}}{2 \varepsilon}\right)^{-1} \leq \frac{\beta}{2 \varepsilon} \prod_{j=N / 2+1}^{N}\left(1+\frac{\beta}{2 \varepsilon N}\right)^{-1} \leq \frac{\frac{\beta}{2 \varepsilon}}{1+\frac{\beta}{4 \varepsilon}} \leq 2, \\
\frac{\beta}{2 \varepsilon} \prod_{j=1}^{N}\left(1+\frac{\beta h_{j}}{2 \varepsilon}\right)^{-1} \leq 2 \prod_{j=1}^{N / 2}\left(1+\frac{\beta h_{j}}{2 \varepsilon}\right)^{-1} \leq C N^{-1},
\end{gathered}
$$

we have

$$
E_{i}^{N} \leq Y_{i} \leq Y_{N / 2} \leq C N^{-1}, \quad i=N / 2, \ldots, N .
$$

From Lemma 1, we have

$$
\left|E_{i}\right| \leq C e^{-\frac{\beta \tau}{\varepsilon}} \leq C N^{-1}, \quad i=N / 2, \ldots, N .
$$

Thus, the proof is complete.

Lemma 7. There exists a constant $C \quad$ such that

$$
\left|E_{i}-E_{i}^{N}\right| \leq C N^{-1} \ln N, \quad i=1, \ldots, N / 2 .
$$

Proof. By Lemma 5, Lemma 1, the mesh generating function (2) and noting that

$$
\sinh \left(\frac{\beta h_{i}}{2 \varepsilon}\right)=\sinh \left(2 N^{-1} \ln N\right) \leq C N^{-1} \ln N, \quad i=1, \ldots, N / 2,
$$


we have

$$
\begin{aligned}
& \left|L^{N}\left(E_{i}-E_{i}^{N}\right)\right| \leq C\left[\varepsilon \int_{x_{i-1}}^{x_{i+1}}\left|E^{\prime \prime \prime}(x)\right| d x+\int_{x_{i-1}}^{x_{i}}\left|E^{\prime \prime}(x)\right| d x\right] \\
\leq & C \int_{x_{i-1}}^{x_{i+1}} \varepsilon^{-2} \exp \left(\frac{\beta x}{2 \varepsilon}\right) d x=C \varepsilon^{-1} \exp \left(\frac{-\beta x_{i}}{2 \varepsilon}\right) \sinh \left(\frac{\beta h_{i}}{2 \varepsilon}\right), \\
\leq & C \varepsilon^{-1} N^{-1} \ln N \prod_{j=1}^{i} \exp \left(\frac{-\beta h_{j}}{2 \varepsilon}\right) \leq C \varepsilon^{-1} N^{-1} \ln N \prod_{j=1}^{i}\left(1+\frac{\beta h_{j}}{2 \varepsilon}\right)^{-1} .
\end{aligned}
$$

Let $\phi_{i}=C_{0} N^{-1} \ln N\left(1+Z_{i}\right)$, from Lemma 4 and 6, we have

$$
\begin{gathered}
L^{N} \phi_{i} \geq C_{0} \varepsilon^{-1} N^{-1} \ln N Z_{i} \geq\left|L^{N}\left(E_{i}-E_{i}^{N}\right)\right|, \\
\phi_{0} \geq 0=\left|E_{0}-E_{0}^{N}\right|, \quad \phi_{N / 2} \geq C_{0} N^{-1} \geq\left|E_{N / 2}-E_{N / 2}^{N}\right|,
\end{gathered}
$$

provided that the constant $C_{0}$ is chosen sufficiently large. So,

$\left|E_{i}-E_{i}^{N}\right| \leq \phi_{i} \leq C N^{-1} \ln N$ by a discrete comparison principle.

Theorem 1. The simple upwind scheme on the Shishkin mesh for the singularly perturbed Robin boundary value problem (1) satisfies:

$$
\left|u_{i}-u_{i}^{N}\right| \leq\left\{\begin{array}{c}
C N^{-1} \ln N, 0 \leq i \leq \frac{N}{2}, \\
C N^{-1}, \frac{N}{2}<i \leq N .
\end{array}\right.
$$

Proof. It is proved by (4), Lemma 6 and 7.

\section{FURTHER RESULTS}

On the Bakhvalov-Shishkin mesh (see [8]):

$$
x_{i}=x\left(t_{i}\right), t_{i}=\frac{i}{N}, i=0,1,2, \ldots, N,
$$

where the mesh generating function is as follows:

$$
x(t)=\left\{\begin{array}{c}
-\frac{2 \varepsilon}{\beta} \ln \left(1-2\left(1-\frac{1}{N}\right) t\right), 0 \leq t \leq \frac{1}{2}, \\
\frac{2 \varepsilon \ln N}{\beta}+2\left(1-\frac{2 \varepsilon \ln N}{\beta}\right)\left(t-\frac{1}{2}\right), \frac{1}{2} \leq t \leq 1,
\end{array}\right.
$$

the simple upwind scheme for solving the singularly perturbed Robin BVP was proved to be uniform first-order convergence (see [4]):

$$
\left|u_{i}-u_{i}^{N}\right| \leq C N^{-1}, 0 \leq i \leq N .
$$

Further, we consider the midpoint upwind scheme for Dirichlet BVP in [9] to be modified for the Robin BVP (1) as follows:

$$
\left\{\begin{array}{c}
L^{N} u_{i}^{N} \equiv-\varepsilon D^{+} D^{-} u_{i}^{N}-b_{i+\frac{1}{2}} D^{+} u_{i}^{N}+c_{i+\frac{1}{2}} \frac{u_{i+1}^{N}+u_{i}^{N}}{2}=f_{i+\frac{1}{2}}, i=1,2, \ldots, N-1, \\
L^{N} u_{N}^{N} \equiv\left(-\frac{\varepsilon}{h_{N}^{2}}+\frac{b_{N}}{2 h_{N}}\right) u_{N-1}^{N}+\left(\frac{2 \varepsilon}{h_{N}^{2}}+c_{N}\right) u_{N}^{N}+\left(-\frac{\varepsilon}{h_{N}^{2}}-\frac{b_{N}}{2 h_{N}}\right) u_{N+1}^{N}=f_{N}, \\
B_{0}^{N} u_{0}^{N} \equiv u_{0}^{N}=A, B_{1}^{N} u_{N}^{N} \equiv u_{N}^{N}+D u_{N}^{N}=B,
\end{array}\right.
$$

where $D u_{N}^{N}=\left(u_{N+1}^{N}-u_{N-1}^{N}\right) /\left(2 h_{N}\right)$. For $i=1,2, \ldots, N-1$, we have

$$
\begin{aligned}
L^{N} u_{i}^{N} & =-\frac{2 \varepsilon}{\left(h_{i+1}+h_{i}\right) h_{i}} u_{i-1}^{N}+\left(\frac{2 \varepsilon}{h_{i+1} h_{i}}+\frac{b_{i+1 / 2}}{h_{i+1}}+\frac{c_{i+1 / 2}}{2}\right) u_{i}^{N}-\left(\frac{2 \varepsilon}{\left(h_{i+1}+h_{i}\right) h_{i+1}}+\frac{b_{i+1 / 2}}{h_{i+1}}-\frac{c_{i+1 / 2}}{2}\right) u_{i+1}^{N} \\
& =-r_{i}^{-} u_{i-1}^{N}+r_{i}^{c} u_{i}^{N}-r_{i}^{+} u_{i+1}^{N},
\end{aligned}
$$

Supposed that $h_{i}<2 \beta_{0} /\|c\|_{\infty}$, we have $r_{i}^{-}, r_{i}^{c}, r_{i}^{+}>0$,

$$
-r_{i}^{-}+r_{i}^{c}-r_{i}^{+}=c_{i+1 / 2} \geq 0, \quad i=1,2, \ldots, N-1 .
$$

For $i=N$, from (8), we have

$$
L^{N} u_{N}^{N} \equiv-\frac{2 \varepsilon}{h_{N}^{2}} u_{N-1}^{N}+\left(\frac{2 \varepsilon}{h_{N}^{2}}+c_{N}+\frac{2 \varepsilon}{h_{N}}+b_{N}\right) u_{N}^{N}=f_{N}+\left(\frac{2 \varepsilon}{h_{N}}+b_{N}\right) B .
$$

The coefficient matrix associated with this $L^{N}$ is also an M-matrix and a discrete comparison principle holds. By using barrier functions, we can obtain the same error estimate on the Shishkin mesh for Robin BVP as that for Dirichlet BVP in the following: $\left|u_{i}-u_{i}^{N}\right| \leq\left\{\begin{array}{c}C N^{-1} \ln N, 0 \leq i \leq \frac{N}{2}, \\ C N^{-2}, \frac{N}{2} \leq i \leq N .\end{array}\right.$

Moreover, we can prove that the midpoint upwind scheme on the Bakhvalov-Shishkin mesh has the uniform convergence: $\left|u_{i}-u_{i}^{N}\right| \leq\left\{\begin{array}{l}C N^{-1}, 0 \leq i \leq \frac{N}{2}, \\ C N^{-2}, \frac{N}{2} \leq i \leq N .\end{array}\right.$

\section{Numerical Examples}

The numerical results in tables 1 and 2 agree with the error estimates for the simple upwind scheme on the Shishkin mesh and the Bakhvalov-Shishkin mesh, denoted by S-S and S-BS, and the midpoint upwind scheme on the Shishkin mesh and the Bakhvalov-Shishkin mesh, denoted by M-S and M-BS. The numerical convergence rates are computed by

$\log _{2}\left(\max \left|u_{i}-u_{i}^{N}\right| / \max \left|u_{i}-u_{i}^{2 N}\right|\right)$ on the coarse part and the fine part, respectively. Denoting the error estimate by $\left|u_{i}-u_{i}^{N}\right| \leq C \sigma(N)$, the constant is computed by $\max \left|u_{i}-u_{i}^{N}\right| / \sigma(N) \cdot$

Problem 1. $\left\{\begin{array}{c}-\varepsilon y^{\prime \prime}-y^{\prime}+y=0,0<x<1, \\ y(0)=0, y(1)+y^{\prime}(1)=1 .\end{array}\right.$

The exact solution of this problem is

$$
\begin{aligned}
& y(x)=\left(e^{m_{1} x}-e^{m_{2} x}\right) /\left[\left(1+m_{1}\right) e^{m_{1}}-\left(1+m_{2}\right) e^{m_{2}}\right], \text { where } \\
& m_{1}, m_{2}=(-1 \pm \sqrt{(1+4 \varepsilon)}) /(2 \varepsilon) .
\end{aligned}
$$




\begin{tabular}{|c|c|c|c|c|c|c|}
\hline$N$ & $i \leq N / 2$ & rate & const & $i \geq N / 2$ & rate & const \\
\hline \multicolumn{7}{|l|}{ S-S: } \\
\hline 64 & 0.0069 & 0.59 & .107 & 0.0077 & 0.98 & .492 \\
\hline 128 & 0.0046 & 0.67 & .122 & 0.0039 & 1.04 & .496 \\
\hline 256 & 0.0029 & 0.77 & .133 & 0.0019 & 0.96 & .498 \\
\hline 512 & 0.0017 & 0.79 & .140 & $9.746 \mathrm{e}-4$ & 1.00 & .499 \\
\hline 1024 & $9.851 \mathrm{e}-4$ & 0.82 & .146 & $4.878 \mathrm{e}-4$ & 1.00 & .500 \\
\hline 2048 & $5.565 \mathrm{e}-4$ & 0.85 & .149 & $2.440 \mathrm{e}-4$ & 1.00 & .500 \\
\hline \multicolumn{7}{|l|}{ S-BS: } \\
\hline 64 & 0.0055 & 0.97 & .353 & 0.0077 & 0.98 & .492 \\
\hline 128 & 0.0028 & 1.00 & .362 & 0.0039 & 1.04 & .496 \\
\hline 256 & 0.0014 & 1.00 & .366 & 0.0019 & 0.96 & .498 \\
\hline 512 & $7.170 \mathrm{e}-4$ & 0.97 & .367 & $9.746 \mathrm{e}-4$ & 1.00 & .499 \\
\hline 1024 & $3.590 \mathrm{e}-4$ & 1.00 & .368 & $4.878 \mathrm{e}-4$ & 1.00 & .500 \\
\hline 2048 & $1.796 \mathrm{e}-4$ & 1.00 & .368 & $2.440 \mathrm{e}-4$ & 1.00 & .500 \\
\hline \multicolumn{7}{|l|}{ M-S: } \\
\hline 64 & 0.0103 & 0.71 & .660 & $1.497 \mathrm{e}-5$ & 2.00 & $.613 \mathrm{e}-1$ \\
\hline 128 & 0.0063 & 0.77 & .809 & $3.744 \mathrm{e}-6$ & 2.00 & $.613 \mathrm{e}-1$ \\
\hline 256 & 0.0037 & 0.82 & .956 & $9.366 \mathrm{e}-7$ & 2.00 & $.111 \mathrm{e}-1$ \\
\hline 512 & 0.0021 & 0.81 & 1.10 & $2.344 \mathrm{e}-7$ & 2.00 & $.985 \mathrm{e}-2$ \\
\hline 1024 & 0.0012 & 0.85 & 1.23 & $5.874 \mathrm{e}-8$ & 1.99 & $.889 \mathrm{e}-2$ \\
\hline 2048 & $6.663 \mathrm{e}-4$ & 0.87 & 1.36 & $1.475 \mathrm{e}-8$ & 1.99 & $.812 \mathrm{e}-2$ \\
\hline \multicolumn{7}{|c|}{ M-BS: } \\
\hline 64 & 0.0055 & 0.97 & .350 & $1.497 \mathrm{e}-5$ & 2.00 & $.613 \mathrm{e}-1$ \\
\hline 128 & 0.0028 & 1.00 & .354 & $3.746 \mathrm{e}-6$ & 2.00 & $.614 \mathrm{e}-1$ \\
\hline 256 & 0.0014 & 1.00 & .356 & $9.373 \mathrm{e}-7$ & 2.00 & $.614 \mathrm{e}-1$ \\
\hline 512 & $6.981 \mathrm{e}-4$ & 1.00 & .357 & $2.347 \mathrm{e}-7$ & 2.00 & $.615 \mathrm{e}-1$ \\
\hline 1024 & $3.496 \mathrm{e}-4$ & 1.00 & .358 & $5.882 \mathrm{e}-8$ & 1.99 & $.616 \mathrm{e}-1$ \\
\hline 2048 & $1.749 \mathrm{e}-4$ & 1.00 & .358 & $1.478 \mathrm{e}-8$ & 1.99 & $.619 \mathrm{e}-1$ \\
\hline
\end{tabular}

Problem 2. $\left\{\begin{array}{c}-\varepsilon y^{\prime \prime}-y^{\prime}=1+2 x, 0<x<1, \\ y(0)=1, y(1)+y^{\prime}(1)=0 .\end{array}\right.$

Its exact solution is given by

$y(x)=\left[(5-4 \varepsilon)-\left(1-\frac{1}{\varepsilon}\right) e^{\frac{-1}{\varepsilon}}+4(\varepsilon-1) e^{\frac{-x}{\varepsilon}}\right] /\left[1-\left(1-\frac{1}{\varepsilon}\right) e^{\frac{-1}{\varepsilon}}\right]-\left(x+x^{2}-2 \varepsilon x\right)$.

TABLE 2. THE ERRORS FOR PROBLEM 2 WITH $\varepsilon=10^{-6}$ AND $\beta=0.5$

\begin{tabular}{lllllll}
\hline$N$ & $i \leq N / 2$ & rate & const & $i \geq N / 2$ & rate & const \\
\hline S-S: & & & & & & \\
16 & 0.8961 & 0.64 & 5.170 & .3594 & 0.97 & 5.75 \\
32 & 0.5749 & 0.63 & 5.310 & .1836 & 0.98 & 5.87 \\
64 & 0.3706 & 0.70 & 5.700 & .0928 & 0.99 & 5.94 \\
128 & 0.2280 & 0.77 & 6.010 & .0466 & 0.99 & 5.97 \\
256 & 0.1336 & 0.80 & 6.170 & .0234 & 1.00 & 5.98 \\
512 & 0.0764 & 0.84 & 6.270 & .0117 & 0.99 & 5.99 \\
S-BS: & & & & & & \\
\hline
\end{tabular}

\begin{tabular}{|c|c|c|c|c|c|c|}
\hline 16 & 0.7088 & 0.90 & 11.30 & .3594 & 0.97 & 5.75 \\
\hline 32 & 0.3796 & 0.93 & 12.10 & .1836 & 0.98 & 5.87 \\
\hline 64 & 0.1986 & 0.97 & 12.70 & .0928 & 0.99 & 5.94 \\
\hline 128 & 0.1017 & 0.98 & 13.00 & .0466 & 0.99 & 5.97 \\
\hline 256 & 0.0515 & 0.99 & 13.20 & .0234 & 1.00 & 5.98 \\
\hline 512 & 0.0259 & 0.99 & 13.30 & .0117 & 0.99 & 5.99 \\
\hline \multicolumn{7}{|c|}{ M-S: } \\
\hline 16 & 0.6784 & 0.56 & 10.9 & $6.081 \mathrm{e}-8$ & 3.37 & $.561 \mathrm{e}-5$ \\
\hline 32 & 0.4614 & 0.54 & 14.8 & $5.902 \mathrm{e}-9$ & 3.92 & $.174 \mathrm{e}-5$ \\
\hline 64 & 0.3174 & 0.69 & 20.3 & $3.895 \mathrm{e}-10$ & 4.13 & $.384 \mathrm{e}-6$ \\
\hline 128 & 0.1974 & 0.73 & 25.3 & $2.231 \mathrm{e}-11$ & 4.18 & $.753 e-7$ \\
\hline 256 & 0.1191 & 0.79 & 30.5 & $1.228 \mathrm{e}-12$ & 2.25 & $.145 \mathrm{e}-7$ \\
\hline 512 & 0.0689 & 0.82 & 35.3 & $2.576 \mathrm{e}-13$ & 2.00 & $.108 \mathrm{e}-7$ \\
\hline \multicolumn{7}{|c|}{ M-BS: } \\
\hline 16 & 0.4455 & 0.84 & 7.13 & $2.070 \mathrm{e}-7$ & 2.16 & $.530 \mathrm{e}-4$ \\
\hline 32 & 0.2488 & 0.91 & 7.96 & $4.646 \mathrm{e}-8$ & 2.52 & $.476 \mathrm{e}-4$ \\
\hline 64 & 0.1320 & 0.95 & 8.45 & $8.078 \mathrm{e}-9$ & 2.74 & $.331 \mathrm{e}-4$ \\
\hline 128 & 0.0681 & 0.98 & 8.71 & $1.205 \mathrm{e}-9$ & 2.87 & $.197 \mathrm{e}-4$ \\
\hline 256 & 0.0346 & 0.99 & 8.85 & $1.649 \mathrm{e}-10$ & 2.92 & $.108 \mathrm{e}-4$ \\
\hline 512 & 0.0174 & 0.98 & 8.93 & $2.175 \mathrm{e}-11$ & 2.98 & $.570 \mathrm{e}-5$ \\
\hline
\end{tabular}

Form the theoretical analysis and the numerical results, we conclude that S-S, S-BS, M-S and M-BS are robust, efficient and $\varepsilon$-uniform convergent.

\section{REFERENCES}

[1] J.J.H. Miller, R.E. O'Riordan, G.I. Shishkin, Fitted Numerical Methods for Singular Perturbation Problems. World Scientific, Singapore, 1996.

[2] H.-G. Roos, M. Stynes, L. Tobiska, Robust Numerical Methods for Singularly Perturbed Differential Equations, Springer-Verlag, Berlin, Heidelberg, 2008.

[3] S. Natesan, N. Ramanujam, An asymptotic-numerical method for singularly perturbed Robin problems-I, Appl. Math. Comput. 126 (2002) 97-107.

[4] Z.-D. Cen, H.-Y. Gao, Uniform convergence analysis of a singularly perturbed Robin problem on a Bakhvalov-Shishkin mesh, J. Zhejiang Univ. (Science Edition) 31 (2004) 373-375 (in Chinese).

[5] R.B. Kellogg, A. Tsan, Analysis of some difference approximations for a singular perturbation problem without turning points, Math. Comput. 32 (1978) 1025-1039.

[6] A.S. Bakhvalov, On the optimization of methods for solving boundary value problems in the presence of boundary layers, $\mathrm{Zh}$. Vychisl. Mat. Mat. Fiz. 9(1969) 841-859 (in Russian).

[7] G.I. Shishkin, Grid approximation of singularly perturbed elliptic and parabolic equations, Second doctorial thesis, Keldysh Institute, Moscow, 1990 (in Russian).

[8] T. Lin $\beta$, An upwind difference scheme on a novel Shishkin-type mesh for a linear convection-diffusion problem, J. Comput. Appl. Math. 110 (1999) 93-104.

[9] M. Stynes, H.-G. Roos, The midpoint upwind scheme, Appl. Numer. Math. 23 (1997) 361-374. 